\title{
Determining Performance Limits for Directed Energy Weapons in Collaborative Active Protections Systems
}

\author{
Graham V. Weinberg ${ }^{a}$ \\ ${ }^{a}$ Defence Science and Technology Group, Joint and Operations Analysis Division, Edinburgh, South \\ Australia, 5111, Australia \\ Email: Graham.Weinberg@dst.defence.gov.au
}

\begin{abstract}
The application of directed energy effectors, and in particular high energy lasers, is of considerable importance for the future land fighting force. Such weapons have the utility for long distance precision strikes against targets, and so merit investigation for potential application in future emerging technology. Collaborative active protection systems is an emerging capability which is designed to provide automatic threat defence for an armoured fighting vehicle team. Performance prediction analysis of such future systems is thus of considerable importance to defence stakeholders.

Recent studies have shown that the performance analysis of such systems can be very complicated, although in some cases this complexity can be reduced. The latter is certainly the situation when the number of threats the combat team is facing is smaller than the number of available countermeasures.

Hence this paper proposes a methodology where this restriction can be eliminated. The key to this is to focus on the likelihood of at least one threat being eliminated, and examining performance prediction curves to assess this likelihood. Once consequence of this is that it becomes necessary to introduce a "rule of thumb" to interpret these performance prediction curves relative to the threat. To illustrate the potential of the methodology, an illustrative example will be analysed. This hypothetical example assumes some generic properties of a high energy laser directed energy weapons, operating in a collaborative active protection system context. It will be shown for this example that threats travelling at speeds significantly smaller than Mach 1 are likely to be countered through the collaborative active protection system, while the rule of thumb establishes that threats exceeding this speed are unlikely to be defeated.
\end{abstract}

Keywords: Armoured fighting vehicles, active protection systems, directed energy weapons, performance limits 


\section{INTRODUCTION}

A combat team of armoured fighting vehicles (AFVs) requires a degree of autonomy in terms of its self-defence against airborne threats. As such, active protection systems (APS) have been designed for this purpose (Meyer (1998); Feickert (2016)). Such systems provide an automatic threat detection mechanism through a suite of sensors, which then utilise tactical information to coordinate effectors on the vehicle. The need to increase defensive effectiveness has resulted in the idea of a collaborative APS (C-APS). Such a system utilises sharing of information between different vehicles and then coordination of effectors to defeat the threats. Some recent examinations of C-APS in the context of interest can be found in Weinberg (2021a) and Weinberg and Kracman (2021). Figure 1 provides an illustration of the combat situation under consideration, where the combat team consists of five vehicles and is facing an attack from three threats.

Of particular interest in the latter cited studies of C-APS has been the application of directed energy weapons (DEWs) for the purpose of vehicle defence (Nielsen (1994)). The DEW which has maximum utility for CAPS application is a high energy laser (HEL), as discussed in Hafften and Stratton (2004). Such a DEW can provide a precision strike against threats commonly enountered in the modern battlefield, as discussed in Weinberg and Kracman (2021). Such threats include drones and rocket propelled grenades (RPGs). The analysis in Weinberg and Kracman (2021) produced a useful expression for the probability of defeat of a target by a HEL DEW, and then applied this to C-APS. However, the methodology in Weinberg and Kracman (2021) became very complicated when the AFV team faced more than a single threat. In the case where the number of threats is known a priori and do not exceed the number of available effectors a somewhat simpler expression for predictive performance can be produced, as discussed and demonstrated in Weinberg (2021b).

The current paper will demonstrate how the issues in these previous studies can be addressed by utilising an alternative mathematical development and interpretation of predicted performance. This approach allows the determination of an approximate "rule of thumb" to determine HEL DEW success under C-APS against a variety of threats. In order to achieve this a number of simplifications are adopted. Firstly, in contrast to the approaches in Weinberg (2021a) and Weinberg and Kracman (2021) the number of threats is assumed to be unknown. Hence the number of arriving threats will be modelled through a stochastic process. The times of detection of threats will be modelled by the process arrival times. This approach allows focus on the benefits of the DEW capability. Secondly, it will become apparent that it is somewhat intractable to produce a useful model for the number of threats defeated by a given time. It turns out to be simpler to instead examine the likelihood that the C-APS system defeats at least one threat. Performance prediction curves based upon this can provide a useful way of determining the limits of HEL DEWs against different threats. Additionally, it will be assumed that the C-APS has the maximum number of effectors available to eliminate the threats. This case, where each AFV is assumed to have a HEL DEW, has been shown in Weinberg (2021a) and Weinberg and Kracman (2021) to provide the maximal probability of defeat of threats. Hence this provides a best case result: as will be shown this best case result will not be effective against certain threats in a particular illustrative example.

The next section introduces the primary mathematical model, where the C-APS performance prediction problem is interpreted within a network of queues framework, in the spirit of Weinberg (2021b).

\section{Mathematical Model}

Throughout the following threats to the AFV will be referred to as particles for convenience. Elements of stochastic processes will be required in the analysis, and so a useful guide for the reader on stochastic processes is Ross (2019). Suppose that particles arrive into the system through a Poisson process with positive rate $\mu$, and let $P(t)$ be the number of particles that have arrived by time $t$. Poisson processes are used for modelling rare events, so in the current context is a plausible assumption (Barbour et al. (1992)).

As particles arrive into the system they are passed to a network of $N$ queues. These queues represent the C-APS effectors, and particles arriving into the system are interpreted as customers to be processed by a queue server (the HEL DEW). These queues process the particles in a first come first serve basis; if the number of pre-processed particles in the system exceeds the number of queues then particles will wait until they are processed by the server in the queue to which they are assigned. Hence these queues are single server with an infinite capacity. It is also assumed that once a particle is placed in a queue it remains there until it is processed, so that movements between queues is not permissible. Thus the C-APS problem is interpreted through a network of queues, and the throughput of these queues can quantify AFV combat team survivability.

To illustrate this, let $M(t)$ be the number of particles in the network of queues at time $t$. Then the random 


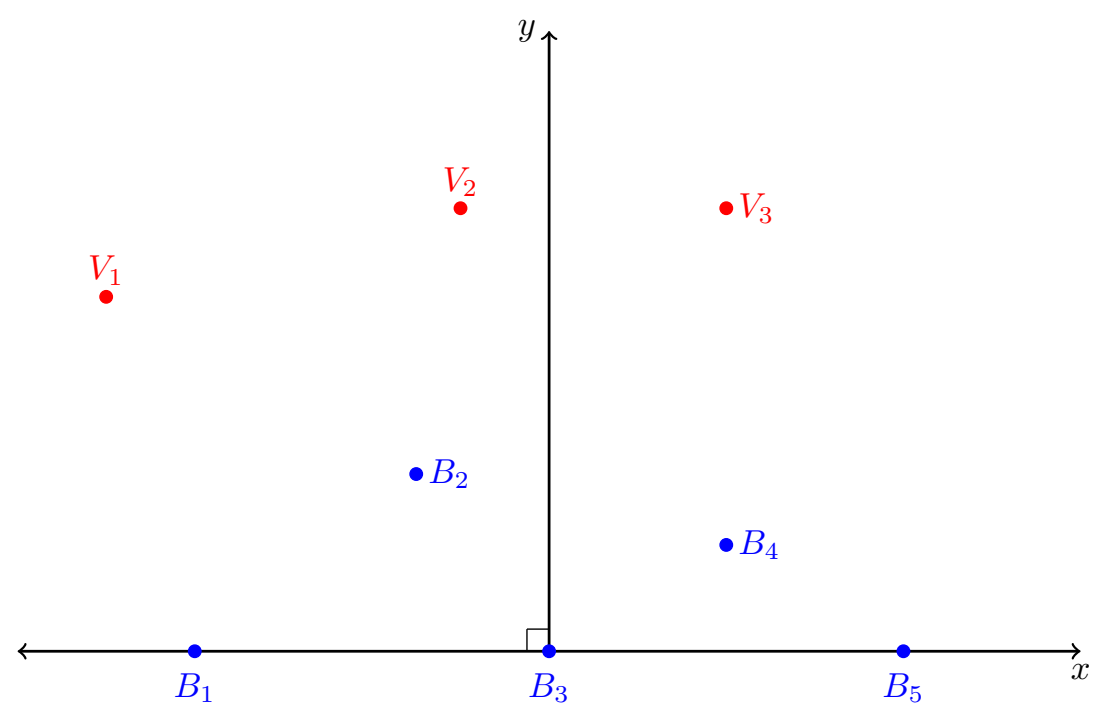

Figure 1. An illustration of the combat scenario under consideration. A combat team consisting of five members $\left(B_{1}, B_{2}, \ldots, B_{5}\right)$ is facing a series of threats $\left(V_{1}, V_{2}, V_{3}\right)$. Throughout the engagement the combat team remains stationary, while the threats are moving.

variable $Z(t)$ defined by

$$
Z(t)=P(t)-M(t)
$$

counts the number of particles processed by the system by time $t$. This in effect counts the number of threats defeated in a C-APS. The random variable $Z(t)$ takes values in the set of non-negative integers. Hence for any integer $k$ it follows by conditioning on the arrival process that

$$
\mathbf{P}(Z(t)=k)=\sum_{w=0}^{\infty} \mathbf{P}(P(t)=w) \mathbf{P}(M(t)=w-k \mid P(t)=w) .
$$

Observe that the random variable $M(t) \geq 0$, since there can only be a nonnegative number of particles in the queueing network. Therefore $(2)$ is equivalent to

$$
\mathbf{P}(Z(t)=k)=\sum_{w=k}^{\infty} \mathbf{P}(P(t)=w) \mathbf{P}(M(t)=w-k \mid P(t)=w) .
$$

Given that $P(t)=w$, where $w$ is a natural number defined through the index in (3), this implies that $w$ particles have appeared by time $t$, and $M(t)=w-k$ is the event that $k$ particles have been processed and $w-k$ still remain in the network of queues.

Since $P(t)=w$ one can suppose that the $w$ particles have arrived at times $\tau_{j}$, for $1 \leq j \leq w$ and such that $\tau_{1}<\tau_{2}<\cdots<\tau_{w}$. Since the arrival process is Poisson, it follows that $\tau_{j}=\tau_{j-1}+\gamma_{j-1}$ where the sequence of $\gamma$ random variables is exponentially distributed with parameter $\mu$ and are independent, for $j \geq 1$ and $\tau_{0}:=0$. Define $\psi_{j}^{(i)}$ to be the service time of particle $j$ when it is in the system and being processed in queue $i$. This service time will depend on the service time of other particles in queue $i$. However, the time it takes for this particular particle to be served by the queue's server can be expressed in terms of the probability that it is defeated by the underlying effector. Suppose that the probability that particle $j$ is eliminated by the server in queue $i$ is $\phi_{j}^{(i)}$, and that $\nu_{j}^{(i)}$ is the time it takes the $i$ th queueing server to process this particle. Then the probability that $\nu_{j}^{(i)}$ exceeds $t$ is equivalent to the probability that particle $j$ is not processed by queue server $i$ by time $t$. Hence

$$
\mathbf{P}\left(\nu_{j}^{(i)}>t\right)=1-\phi_{j}^{(i)} .
$$

Consequently (4) characterises the time it takes for the queue server to process the particle in the given queue. Note that $\psi_{j}^{(i)} \geq \nu_{j}^{(i)}$, and the former variable must include the time each particle spends in queue $i$. 
The conditional probability $\mathbf{P}(M(t)=w-k \mid P(t)=w)$ can in principle be expressed in terms of the particle arrival and service time, but can be somewhat complicated in practice. However it is worth noting that performance analysis can be undertaken on the basis of whether no or at least one particle has been processed by the system. Hence consider ( 3 ) when $k=0$, that is, the probability that $Z(t)$ is zero:

$$
\mathbf{P}(Z(t)=0)=\sum_{w=0}^{\infty} \mathbf{P}(P(t)=w) \mathbf{P}(M(t)=w \mid P(t)=w) .
$$

Now the probability that $M(t)$ is zero, under the condition that $P(t)$ is zero, is unity. This is because of the fact that if no particles have appeared, then there are no particles in the network of queues. For any $j \leq N$ the probability that $M(t)=j$ given $P(t)=j$ is the probability that the first $j$ particles have not been processed by the queueing network by time $t$. Hence

$$
\mathbf{P}(M(t)=j \mid P(t)=j)=\mathbf{P}\left(\tau_{1}+\nu_{1}>t, \tau_{2}+\nu_{2}>t, \ldots, \tau_{j}+\nu_{j}>t\right),
$$

where it is being assumed that particles are assigned to the queues systematically, since there will be more queues free than the total number of particles that have arrived. In addition, $\nu_{j}$ is the time particle $j$ spends in queue $j$, which is the server service time alone in the queue.

Observe that when the number of particles present exceeds $N$, the probability that none of these particles have been processed implies that the original set of $N$ are still being processed by the queue servers, while the excess of particles are waiting in the network of queues. Hence

$$
\mathbf{P}(M(t)=j+N \mid P(t)=j+N)=\mathbf{P}\left(\tau_{1}+\nu_{1}>t, \tau_{2}+\nu_{2}>t, \ldots, \tau_{N}+\nu_{N}>t\right) .
$$

The inherent advantage of (7) is that it does not require consideration of the waiting times of particles exceeding the total service capacity of $N$ in the network of queues.

Hence an application of (6) and (7) to (5) yields

$$
\begin{aligned}
\mathbf{P}(Z(t)=0)= & \sum_{w=0}^{N-1} \mathbf{P}(P(t)=w) \mathbf{P}\left(\tau_{1}+\nu_{1}>t, \ldots, \tau_{w}+\nu_{w}>t\right) \\
& +\sum_{w=N}^{\infty} \mathbf{P}(P(t)=w) \mathbf{P}\left(\tau_{1}+\nu_{1}>t, \ldots, \tau_{N}+\nu_{N}>t\right) \\
= & \mathbf{P}(P(t)=0)\left[1-\mathbf{P}\left(\tau_{1}+\nu_{1}>t, \ldots, \tau_{N}+\nu_{N}>t\right)\right] \\
& +\sum_{w=1}^{N-1} \mathbf{P}(P(t)=w) \times \\
& \left\{\mathbf{P}\left(\tau_{1}+\nu_{1}>t, \ldots, \tau_{w}+\nu_{w}>t\right)-\mathbf{P}\left(\tau_{1}+\nu_{1}>t, \ldots, \tau_{N}+\nu_{N}>t\right)\right\} \\
& +\mathbf{P}\left(\tau_{1}+\nu_{1}>t, \ldots, \tau_{N}+\nu_{N}>t\right) .
\end{aligned}
$$

In view of the fact that $\mathbf{P}(Z(t) \geq 1)=1-\mathbf{P}(Z(t)=0)$, one can hence examine the likelihood of at least one particle being defeated by time $t$. This can be used to produce a rule of thumb to facilitate the performance analysis of schemes such as C-APS in the presence of challenging threats. The next section will illustrate this procedure.

\section{Application to C-APS With HEL DEW}

In the following a hypothetical application is produced to illustrate the application of the above results, where all DEW operational parameters are chosen arbitrarily and propagation characteristics have been based upon those reported in Spangle et al. (2005) and Spangle et al. (2011). Suppose that each effector (or queue server) is a HEL DEW, operating at a wavelength of $\lambda=1.045 \mu \mathrm{m}$ with output power of $30 \mathrm{~kW}$. Thus each DEW in the C-APS is homogeneous. Then the probability of disruption of a target, as derived in Weinberg and Kracman (2021), is given by

$$
\phi_{j}^{(i)}(t)=\frac{\mu_{\tau}}{1-e^{-\mu_{\tau} t}} \int_{0}^{t} e^{-\mu_{\tau} s} e^{-1.8194 \mu_{\sigma} U\left[\int_{t-s}^{t}\left(R_{j}^{(i)}(x)\right)^{-2} d x\right]^{-1}} d s,
$$


where $R_{j}^{(i)}(t)$ is the distance between the $j$ th particle and the $i$ th effector at time $t$, the reciprocal of $\mu_{\tau}$ is the expected DEW dwell time on the target to cause a disruption and the reciprocal of $\mu_{\sigma}$ is the expected vulnerability area of the target. The HEL DEW disruption threshold of the target is $U$, which is the HEL power level on the target to cause a thermal effect, and is assumed to be 10 Watts per square metre. Further details of the derivation and assumptions adopted for the HEL DEW can be sourced from Weinberg and Kracman (2021). For the purposes of this paper the average dwell time has been selected to be 3 seconds and the expected vulnerability area is 1 square metre. Again, these figures have been chosen for illustrative purposes only. Expression (9) can be applied to (4) to determine the corresponding $\nu_{j}$ in (8). This will be done through a simulation method.

The Poisson process arrival rate is taken to be $\mu=1$. Then the $\tau_{j}$ in (7) are sums of exponential random variables with unity mean. It will be assumed that the particle arrival times and the queue server times are independent. Based upon these considerations a Monte Carlo scheme can be applied to evaluate the expressions in (8), with $10^{4}$ runs.

For simplicity $N$ has been selected to be three for the following example, and Figure 2 provides a plot of the probability of at least one particle defeated as a function of time. In each case it is assumed that it takes $T=120$ seconds for the threats to reach their targets if not disrupted. The five cases in Figure 2 correspond to different speeds of the threats, which are assumed to be on a direct course to their intended target. Case 1 is for the situation where each of the threats in the network of queues have speeds of 30 metres per second. Case 2 increases one of these speeds to 50 while the other two are 100 metres per second. Case 3 is for the situation where one particle is travelling at 150 metres per second while the other two travel at 300 metres per second. Case 4 illustrates the scenario where each particle is travelling at the speed of sound (Mach 1, 343 metres per second). Finally, Case 5 provides an illustration of performance with hypersonic speeds. Here the three particles travel at Mach 5 (1715 metres per second).

Given the differences in speed, and the fact that the particles are assumed to reach the intended targets in 120 seconds, the distances travelled by each particle will differ considerably between cases. This is important to note when interpreting Figure 2

Consider Case 1: the three threats will reach their intended targets after travelling a distance of $3.6 \mathrm{~km}$. The plot corresponding to Case 1 in Figure 2 demonstrates that from time approximately 20 seconds, the likelihood of at least one threat being defeated is almost certain. After 20 seconds the threats are $3 \mathrm{~km}$ from their intended target, and so such a result is consistent with the observations in Weinberg and Kracman (2021).

Another important consideration from Case 1 is the resultant "flatlining" of the probability to unity. This is an important characteristic for a successsful DEW because it implies that there will be sufficient dwell time to cause the disruption of the particles. In this example a sufficient dwell time can be taken to be the assumed mean dwell time in the model, which is 3 seconds. The model of HEL DEW effects (9) implies that at the upper limit of $t=T=120$ the disruption probability is unity; however this is only going to be the case in reality if there is sufficient DEW dwell time, and the flatlining demonstrates this to be likely in Case 1.

Cases 2 and 3 also demonstrate this to be the situation, and one can deduce there will be sufficient dwell time to eliminate at least one threat. However in Cases 4 and 5 there is a completely different situation. Consider the extreme Case 5: the threats are travelling such that in their last 10 seconds of travel they would traverse a distance of 188, 650 metres. The plot corresponding to Case 5 in Figure 2 indicates that there will be insufficient opportunity for the illustrative DEW's dwell time on the target, despite the fact that the model suggests the probability of at least one being defeated is climbing up to almost unity.

Thus a rule of thumb, or sine qua non to guarantee C-APS effectiveness, can be deduced when analysing HEL DEW performance using the techniques introduced in this paper. When plotting the probability of at least one particle being defeated as a function of time, an absence of flatlining near unity for a sufficient period of the threat travel time (such as expected dwell time) will indicate that although the model may limit to unity it is unlikely that there is sufficient DEW dwell time to defeat threats.

Threat speed obviously plays a significant role in the analysis here. For the given hypothetical DEW specifications utilised above one can determine drones and RPGs are suitable threats for the system to defeat, but hypersonic threats will be almost impossible to defeat. 


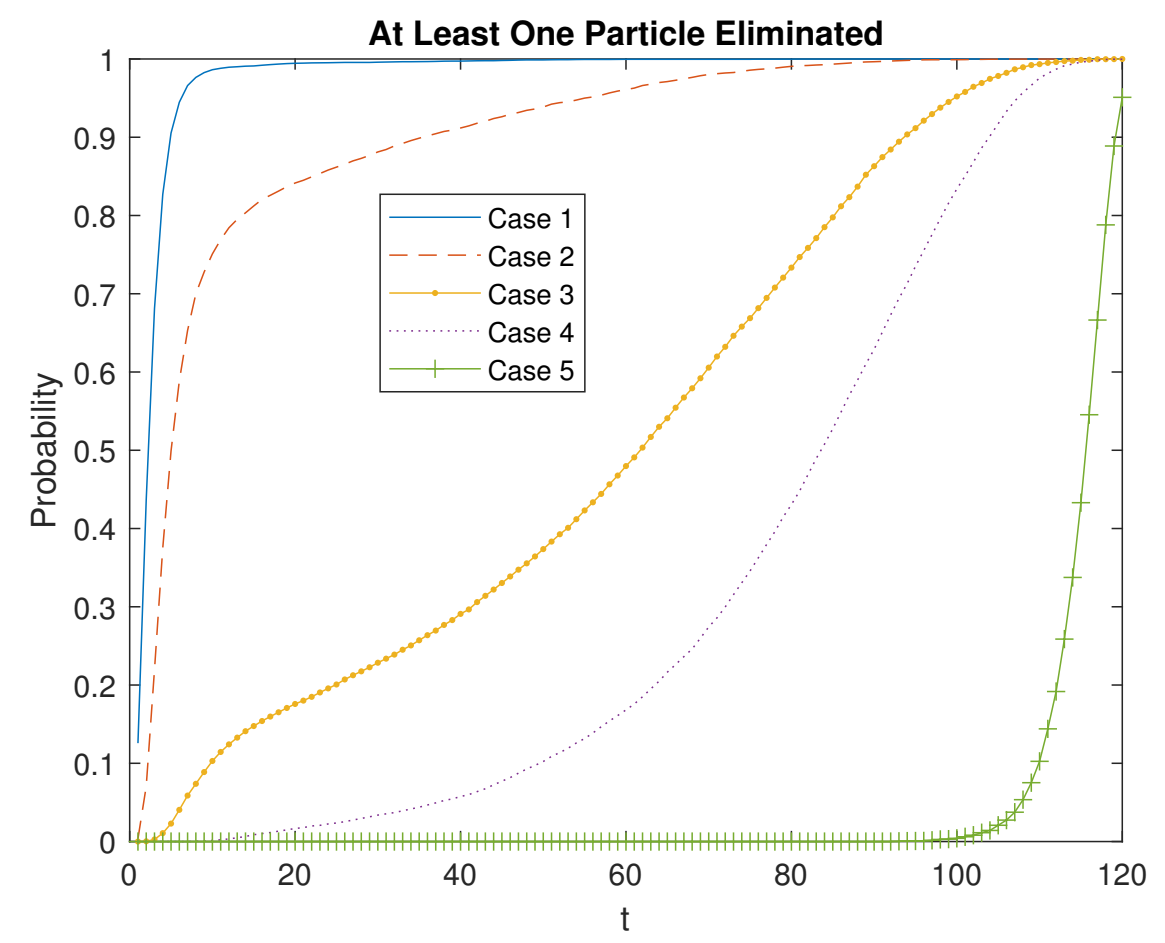

Figure 2. The probability of at least one threat or particle being defeated by time $t$, in the case where the number of effectors is $N=3$ and the arrival process is Poisson with mean rate unity. The threat speeds vary from 30 metres per second (Case 1) to Mach 5 (Case 5).

\section{Conclusions}

The purpose of this paper was to provide a mathematical model for performance prediction of C-APS when defence is provided by a HEL DEW. A rule of thumb was derived to interpret plots of the probability of at least one threat being defeated, as a function of time. A Poisson process was used to model the arrival of threats, interpreted as particles appearing in a network of queues. HEL effectors were interpreted as the queue servers in this network.

The rule of thumb mandates that the correct analysis of the performance prediction curves involves investigating the flatlining to unity probability, which implies that there will be sufficient dwell time for the threats to be defeated by the HEL DEW. The approach in this paper can be applied to any HEL DEW, operating in a C-APS setting, to assess its performance against a variety of different threats by adapting (9) to produce suitable performance curves, and applying this rule of thumb.

\section{ACKnOWledgements}

This paper was motivated by discussions with Mr Nick Kempt and Dr Ashley Stephens during a presentation on performance of C-APS systems at DSTG. The comments of the two anonymous reviewers were appreciated and improved the paper considerably. 


\section{REFERENCES}

Barbour, A. D., L. Holst, and S. Janson (1992). Poisson Approximation. Oxford Science Publications.

Feickert, A. (2016). Army and marine corps active protection systems (APS) efforts. In United States Congressional Research Service Report.

Hafften, M. and R. Stratton (2004). High energy laser weapon integration with ground vehicles. In NATO Report presented to RTO AVT Symposium, Volume RTO-MP-AVT-108.

Meyer, T. J. (1998). Active protective systems: Impregnable armor or simply enhanced survivability? In Armor, Volume May-June.

Nielsen, P. E. (1994). Effects of Directed Energy Weapons. Washington: National Defence University.

Ross, S. M. (2019). Introduction to Probability Models. Academic Press.

Spangle, P., J. Penano, and B. Hafizi (2005). Optimum Wavelength and Power for Efficient Laser Propagation in Various Atmospheric Environments. Naval Research Laboratory Report, NRL/MR/6790-05-8907.

Spangle, P., J. Penano, and B. Hafizi (2011). Beam Combining and Atmospheric Propagation of High Power Lasers. Naval Research Laboratory Report, NRL/MR/6790-11-9371.

Weinberg, G. V. (2021a). Quantification of combat team survivability with high power RF directed energy weapons. Progress In Electromagnetics Research M 102, 1-11.

Weinberg, G. V. (2021b). A queueing theoretic approach for performance prediction of collaborative active protection systems. In MODSIM2021 Conference Proceedings.

Weinberg, G. V. and M. M. Kracman (2021). Armoured fighting vehicle team performance prediction against missile attacks with directed energy weapons. ArXiv Preprint, arXiv:2106.14381v1. 